Short Title: Nuclear position affects pollen ROS and calcium response

Author for contact: Iris Meier

The Ohio State University, 520 Aronoff Laboratory,

Columbus, $\mathrm{OH} 43210$

meier.56@osu.edu, (614) 2928323

\title{
Article Title: Vegetative nuclear positioning is required for calcium and ROS signaling in Arabidopsis pollen tubes
}

Morgan Moser ${ }^{1+}$, Andrew Kirkpatrick ${ }^{1+}$, Norman Reid Groves ${ }^{1,3}$, and Iris Meier ${ }^{1,2,3 *}$

${ }^{1}$ Department of Molecular Genetics, The Ohio State University, Columbus, $\mathrm{OH}, \mathrm{USA}$

${ }^{2}$ Center for RNA Biology, The Ohio State University, Columbus, OH, USA

${ }^{3}$ Center for Applied Plant Science, The Ohio State University, Columbus, OH, USA

* Address correspondence to meier.56@osu.edu

+ These authors contributed equally.

Summary: Mutants deficient in outer nuclear membrane proteins display defects in reactive oxygen species-induced pollen tube burst and nuclear $\mathrm{Ca}^{2+}$ signatures that correlate with the position of the vegetative nucleus.

Author Contributions: M.M., A.K., and I.M. designed experiments; M.M. and A.K. performed experiments; M.M., A.K., N.R.G., and I.M. developed methods, interpreted experiments, wrote, and edited the manuscript. I.M. supervised the work and provided funding.

Funding: This work was supported by a grant from the National Science Foundation to I.M. (NSF-1613501). 


\section{Abstract}

Efficient transport and delivery of sperm cells (SCs) is vital for angiosperm plant fertility. In Arabidopsis thaliana, SCs are transported through the growing pollen tube by a connection with the vegetative nucleus (VN). During pollen tube growth, the VN leads the way and maintains a fixed distance from the pollen tube tip, while the SCs lag behind the VN. Upon reception at the ovule, the pollen tube bursts and the SCs are released for fertilization. In pollen tubes of Arabidopsis mutants wit12 and wifi, deficient in the outer nuclear membrane component of a plant LINC complex, the SCs precede the VN and the VN falls behind. Subsequently, pollen tubes frequently fail to burst upon reception. In this study, we sought to determine if the pollen tube reception defect observed in wit12 and wifi is due to decreased sensitivity to reactive oxygen species (ROS). Here we show that wit12 and wifi are hyposensitive to exogenous $\mathrm{H}_{2} \mathrm{O}_{2}$, and that this hyposensitivity is correlated with decreased proximity of the VN to the pollen tube tip. Additionally, we report the first instance of nuclear $\mathrm{Ca}^{2+}$ spikes in growing pollen tubes, which are disrupted in the wit12 mutant. In the wit12 mutant, nuclear $\mathrm{Ca}^{2+}$ spikes are reduced in response to exogenous ROS, but these spikes are not correlated with pollen tube burst. This study finds that VN proximity to the pollen tube tip is required for both response to exogenous ROS, as well as internal nuclear $\mathrm{Ca}^{2+}$ fluctuations. 


\section{Introduction}

Angiosperm fertilization requires delivery of the non-motile sperm cells (SCs) to the ovules by pollen tubes. This process is guided by a variety of interactions between the pollen tube and the surrounding sporophytic and finally gametophytic female tissue. While significant progress has been made towards understanding the underlying malefemale crosstalk and the signaling components involved, the final step, pollen tube reception and termination, still is among the least understood (Dresselhaus and Franklin-Tong, 2013; Higashiyama and Yang, 2017; Johnson et al., 2019).

Pollen tube reception at the ovule involves intricate interactions between the pollen tube and the synergid cells, leading to pollen tube growth arrest and burst, and the release of the SCs (Kessler and Grossniklaus, 2011; Dresselhaus and Franklin-Tong, 2013).

Several signaling components of this step on the female side have been identified (For review see (Johnson et al., 2019)). Loss of function mutants of the synergid cell surface receptor kinase FERONIA lead to failed WT pollen tube burst upon arriving at a fer mutant ovule, and subsequent pollen tube overgrowth (Escobar-Restrepo et al., 2007). FERONIA is required for an increase in reactive oxygen species (ROS) production by the synergid cells upon pollen tube arrival and a synergid calcium $\left(\mathrm{Ca}^{2+}\right)$ fluctuation signal that precedes a corresponding $\mathrm{Ca}^{2+}$ spike in the pollen tube tip prior to burst (Iwano et al., 2012; Ngo et al., 2014). In vitro-applied ROS leads to pollen tube growth arrest and rupture in a $\mathrm{Ca}^{2+}$-dependent manner, suggesting pollen tube-synergid crosstalk involves a synergid-triggered $\mathrm{Ca}^{2+}$-ROS signal that leads to pollen tube termination and sperm cell release (Duan et al., 2014).

How signals from the synergids are perceived by the pollen tube is less well understood. Two FERONIA-related proteins, ANXUR1 and ANXUR2, are specifically expressed in pollen, and the double mutant shows premature growth arrest and burst, but whether they are involved in signal reception by the pollen tube is not known (Boisson-Dernier et al., 2009). A second pair of pollen tube receptors (BUPS1 and BUPS2) has a similar function, protecting the pollen tube from premature burst. BUPS1 and BUPS2 bind both pollen-expressed and female-expressed Rapid Alkalinization Factor (RALF) peptide ligands, and interaction with female-expressed RALF34 releases 
the protection from burst by BUPS1 and BUPS2 (Ge et al., 2017). Thus, an interplay between ovule-derived and pollen-derived peptide ligands might enable the pollen tube to respond to the synergid environment created by FERONIA and related pathways (Ge et al., 2017)

During pollen germination and subsequent pollen tube growth, the two SCs are physically connected with the pollen tube nucleus ("vegetative nucleus", VN), and collectively named the male germ unit (MGU) (Borg et al., 2009). As the pollen tube grows, the MGU maintains a fixed distance from the advancing pollen tube tip, with the VN leading the SCs ( Li et al., 2018). The fact that the VN and SCs migrate as a unit has been proposed to be important for efficient movement of the SCs to the ovule (Russell and Cass, 1981; Dumas et al., 1985; McCue et al., 2011). A mutant that transports only the $\mathrm{VN}$ is phenotypically near-normal, including pollen tube rupture after entering the ovule (Zhang et al., 2017), suggesting that reception and termination signaling can proceed in the absence of the SCs.

In contrast, mutants that transport the SCs, but lead to a partial loss of the VN at the pollen tube tip have defects in pollen tube termination (Zhou and Meier, 2014). The mutations causing this effect are in genes coding for the plant outer nuclear membrane (ONM) proteins WIP (WIP1, WIP2, and WIP3) and WIT (WIT1 and WIT2), the components of a plant LINC complex (Zhou et al., 2012). LINC complexes are protein complexes spanning the inner nuclear membrane (INM) and ONM, thus connecting the nucleus to cytoplasmic components, such as the cytoskeleton (Starr and Fridolfsson, 2010). The core of LINC complexes is composed of the ONM Klarsicht/ANC-1/Syne homology (KASH) proteins, with interact with the INM Sad1 and UNC-84 (SUN) proteins in the nuclear envelope lumen. In animals, LINC complexes are often involved in nuclear movement and nuclear positioning (Starr and Fridolfsson, 2010). Plant LINC complexes contain conserved SUN proteins but have a divergent complement of KASH proteins (Graumann et al., 2010; Zhou et al., 2012). The WIP and WIT protein families form a LINC complex with Arabidopsis SUN1 and SUN2. This complex binds the Myosin XI-i motor protein and is involved in moving nuclei in root and leaf cells (Tamura et al., 2013). In pollen, the WIT-WIP-SUN complex is involved in male fertility. Loss of 
either the WIP or WIT protein family, as well as loss of both, results in a $50 \%$ reduction in seed set and a reversed order within the MGU upon emergence from the pollen grain (Zhou and Meier, 2014). While pollen tube growth is not significantly altered, the MGU distance from the growing pollen tube tip is increased (Zhou and Meier, 2014).

Frequently, the $\mathrm{VN}$ is not observed at the pollen tube tip, and the corresponding pollen tubes either stall at the entrance to an ovule or continue to grow past the synergids but remain intact (Leydon et al., 2013; Zhou and Meier, 2014). How the requirement for the WIT-WIP-SUN complex, and, by extension, the tip-located VN, relates to the known steps of pollen tube reception and termination signaling is not known.

Here, we hypothesized that the WIT-WIP-SUN LINC complex is required for ROSinduced pollen tube rupture. We report a decrease in $\mathrm{Ca}^{2+}$-dependent ROS-induced pollen tube rupture of wip and wit mutants grown semi-in vivo. The hyposensitivity to ROS correlates with an increased distance of the VN from the pollen tube tip. In addition, we provide a first report of nuclear $\mathrm{Ca}^{2+}$ spiking in a gametophytic nucleus and show the patterns during growth and ROS-mediated pollen tube burst are altered in the mutants.

\section{Results and Discussion}

This study uses two previously described Arabidopsis mutant lines "wit12" (T-DNA insertions in WIT1 and WIT2) and "wifi" (T-DNA insertions in WIP1, WIP2, WIP3, WIT1, and WIT2) that have defects in MGU trafficking and pollen tube rupture (Zhou and Meier, 2014). Pollen tubes were tested for their sensitivity to external application of hydrogen peroxide $\left(\mathrm{H}_{2} \mathrm{O}_{2}\right)$ by performing semi-in vivo pollen tube rupture assays at 5 hours post pollination (hpp) and at $7 \mathrm{hpp}$, as described previously (Duan et al., 2014). Pollen tube rupture events were defined by the appearance of cytoplasmic material at the end of the pollen tube (Fig. 1A, B), as described before (Duan et al., 2014). At 5 hpp, approximately $45 \%$ of Columbia- 0 wild type (WT) pollen tubes treated with $\mathrm{H}_{2} \mathrm{O}_{2}$ burst, corroborating prior studies (Duan et al., 2014). In contrast, a reduced number of rupture events was observed in wit12 and wifi pollen, with a burst frequency of $37 \%$ and $34 \%$ respectively (Fig. 1C). 
When the experiment was extended by another 2 hours ( $7 \mathrm{hpp}$ ), efficiency of burst dropped in WT pollen tubes to $35 \%$ but dropped substantially more in wit12 $(16 \%)$ and wifi (18\%). At both $5 \mathrm{hpp}$ and $7 \mathrm{hpp}$, the two mutants behaved similarly (Fig. 1C, D). This suggests that semi-in vivo pollen tube burst in wit12 and wifi is hyposensitive to external ROS, and that this effect increases over time. When pollen tubes were grown on low calcium $\left(\mathrm{Ca}^{2+}\right)$ media and treated with the $\mathrm{Ca}^{2+}$ channel inhibitor gadolinium(III) chloride prior to the application of $\mathrm{H}_{2} \mathrm{O}_{2}$, all three lines behaved similarly with few to no rupture events observed (Figure 1D). In addition, we analyzed time to response of pollen tubes rupture within 10 minutes after addition of $\mathrm{H}_{2} \mathrm{O}_{2}$ (Figure 1E). The data show that there was an overall delay in response in the wit12 pollen tube population compared to WT.

Together, these data show that wit12 and wifi are similarly hyposensitive to ROSinduced pollen tube rupture, that this hyposensitivity increases over time post pollination, and that while pollen tubes burst less frequently in wit12 and wifi, the burst still depends on $\mathrm{Ca}^{2+}$.

Because mutations in WIP and WIT affect the mobility of the VN during pollen tube growth, we tested whether the nuclear position plays a role in ROS response. Because wit12 and wifi pollen tubes showed a comparable response to ROS, as well as comparable male fertility defects (Zhou and Meier, 2014), we proceeded with the wit12 mutant only. WT and wit12 pollen tubes concurrently expressing the VN (Ub10pro::NLS$m$ Cherry) and SC (MGH3pro::GFP) fluorescent markers were germinated using the semi-in vivo method and the nuclear position was examined. In WT, the VN remained at a constant distance of approximately $65 \mu \mathrm{m}$ from the pollen tube tip, regardless of when the pollen tubes were examined (Figure 1F, 1G). By contrast, at $5 \mathrm{hpp}$ the $\mathrm{VN}$ of wit12 pollen tubes was positioned, on average, $118 \mu \mathrm{m}$ from the pollen tube tip (Figure $1 \mathrm{H}$, $1 \mathrm{~J}$ ). This distance was further increased to an average of $157 \mu \mathrm{m}$ at $7 \mathrm{hpp}$ (Figure 1I, $1 \mathrm{~J})$. The separation between VN and SCs in wit12 also increased between $5 \mathrm{hpp}$ and 7 hpp (Figure 1J).

In addition, we noted that wit12 nuclei appeared less elongated than WT nuclei, which were thin and highly stretched (Figure $1 \mathrm{~K}, 1 \mathrm{M}$ ). Circularity index was calculated for WT 
and wit12 nuclei, to determine if a quantifiable difference in circularity exists. When compared to WT, wit12 nuclei are shorter and more circular (Figure 1L, 1M). Taken together, these data indicate a correlation between $\mathrm{VN}$-tip distance and responsiveness to exogenous ROS in the pollen tube rupture mechanism.

A relationship between position of the nucleus and the strength of a signaling pathway has been shown in animals, for example in the notch signaling pathway (Del Bene et al., 2008). In this case, a diffusible compound is involved and the signal reaching the nucleus is dampened by increased distance. Reception of the signal requires a LINC complex for nuclear movement, a shared similarity with the wit12 and wifi mutants described here (Del Bene et al., 2008; Zhou and Meier, 2014). In Arabidopsis pollen, mutants in a family of transcription factors (MYB97/101/120) show similar pollen tube reception defects as wit12 and wifi mutants (Leydon et al., 2013; Liang et al., 2013). A diffusible signal might thus be upstream of the VN gene expression profile required for late stages of pollen tube growth.

As an obvious candidate for such a signal, we tested whether the cytoplasmic $\mathrm{Ca}^{2+}$ signatures of growing pollen tube tips were impaired. Transgenic lines expressing the cytoplasmic $\mathrm{Ca}^{2+}$ sensor R-GECO1 under the pollen-specific promoter Late Anther Tomato 52 (LAT52) (Eyal et al., 1995) were used in time-lapse imaging of WT and wit12 pollen tubes at $7 \mathrm{hpp}$ (Figure 2A, 2B). Transgenic pollen was germinated using the semi-in vivo method. The fluorescence signatures were measured at the tips of growing WT and wit12 pollen tubes using a region of interest (ROI) based analysis method in the NIS Elements analysis software (see Materials and Methods). Qualitatively, both WT and wit12 pollen tubes exhibited a dynamic fluorescence pattern (Figure 2C, 2D) consistent with what has been previously reported for growing Arabidopsis pollen tubes (Iwano et al., 2009). Variability in observed fluorescence intensities between WT and wit12 may be due to different insertional sites between transgenic lines. To determine differences in $\mathrm{Ca}^{2+}$ fluctuations between WT and wit12, the traces were quantified in two ways, by counting the number of peaks after setting a threshold value (Figure $2 \mathrm{E}$ ), and by calculating the average standard deviation of all traces (Figure 2F) (see Materials and Methods). WT and wit12 exhibited similar peak numbers, with an average of 7 
peaks within 10 minutes of imaging (Figure 2E). The standard deviation between WT and wit12 was also similar (Figure $2 \mathrm{~F}$ ). These data suggest that the cytoplasmic $\mathrm{Ca}^{2+}$ signatures at the pollen tube tip of wit12 are not significantly altered during elongation.

A change in the $\mathrm{Ca}^{2+}$-dependent capacity for ROS-induced rupture that correlates with nuclear position might suggest a nuclear $\mathrm{Ca}^{2+}$ signaling step. Several examples for nuclear $\mathrm{Ca}^{2+}$ signaling have been reported in plants, ranging from plant-microbe interactions to development (Capoen et al., 2011; Charpentier et al., 2016; Leitão et al., 2019). To observe nuclear $\mathrm{Ca}^{2+}$, we generated transgenic WT and wit12 plants containing the VN-localized $\mathrm{Ca}^{2+}$ sensor NLS-YC3.6, driven by the LAT52 promoter (Figure 3A-3C). WT nuclei exhibited clear $\mathrm{Ca}^{2+}$ fluctuations at $7 \mathrm{hpp}$, with an average of 3.5 peaks within 10 minutes of imaging (Figure 3D, 3F). In contrast, the $\mathrm{Ca}^{2+}$ signature observed in elongating wit12 pollen tubes exhibited one of two distinct patterns. In the first case, the number of $\mathrm{Ca}^{2+}$ fluctuations were reminiscent of those observed in WT (Figure 3E). In the second case, the number of $\mathrm{Ca}^{2+}$ fluctuations were reduced compared to WT or no fluctuations were observed (Figure 3E).

We next tested if the reduction in nuclear $\mathrm{Ca}^{2+}$ fluctuations observed in wit12 was dependent on the distance of the VN to the pollen tube tip. For each 10-minute movie generated, the distance between the VN and tip was also recorded. The WT nuclei were positioned between 50 and $100 \mu \mathrm{m}$ from the tip (Figure 3F, 3G), consistent with data presented in Figure 1J. VNs in wit12 were distributed over a range of tip distances, but never below $100 \mu \mathrm{m}$. wit12 VNs located between $100 \mu \mathrm{m}$ and $200 \mu \mathrm{m}$ from the pollen tube tip exhibited a more WT-like $\mathrm{Ca}^{2+}$ signature, although the average frequency of peaks per 10 minutes was reduced to 2 peaks (Figure 3F, 3G). wit12 VNs located further than $200 \mu \mathrm{m}$ from the pollen tube tip exhibited a more disrupted $\mathrm{Ca}^{2+}$ signature, on average showing only 1 peak (Figure 3F, 3G). These data show that there are nuclear $\mathrm{Ca}^{2+}$ fluctuations during pollen tube growth and that the frequency of fluctuations is correlated with the proximity of the VN to the pollen tube tip.

Nuclear $\mathrm{Ca}^{2+}$ fluctuations have been described as compounds of signal transduction pathways in a variety of biological systems, notably during root symbioses (Capoen et al., 2011; Liang et al., 2014; Charpentier et al., 2016). After nodulation (Nod) or 
mycorrhizal (Myc) factor perception, pronounced $\mathrm{Ca}^{2+}$ oscillations in and around the nucleus are observed, which are required for the transcriptional response of the host plant (Charpentier, 2018). Examples of nuclear $\mathrm{Ca}^{2+}$ spiking were also found in relation to biotic and abiotic stress as well as root development; however, in these cases single or few spikes were observed instead of pronounced oscillations (Charpentier, 2018; Leitão et al., 2019).

The $\mathrm{Ca}^{2+}$ signal signatures observed here are similar to those observed after biotic or abiotic stress (Lecourieux et al., 2005; Kelner et al., 2018). This response is dampened in the wit12 mutant. Furthermore, wit12 mutant pollen tubes showing the greatest reduction in $\mathrm{Ca}^{2+}$ spiking have the greatest distance between VN and pollen tube tip. It has been shown that plant nuclei can generate their own $\mathrm{Ca}^{2+}$ signals, independent of changes in cytoplasmic $\mathrm{Ca}^{2+}$, and that a simple increase of the extranuclear $\mathrm{Ca}^{2+}$ concentration is not sufficient to trigger $\mathrm{Ca}^{2+}$ increases in isolated nuclei (Pauly et al., 2000; Mazars et al., 2009). It is therefore unlikely that the nuclear $\mathrm{Ca}^{2+}$ spikes in the pollen tube simply reflect cytoplasmic $\mathrm{Ca}^{2+}$ fluctuations by passive $\mathrm{Ca}^{2+}$ influx. Instead, a cytoplasmic $\mathrm{Ca}^{2+}$ signal could be required to directly or indirectly activate a nuclear $\mathrm{Ca}^{2+}$ signal (Charpentier, 2018), and distance from the tip could therefore dampen this response.

Finally, we examined how the cytoplasmic and nuclear $\mathrm{Ca}^{2+}$ signatures changed in response to $\mathrm{H}_{2} \mathrm{O}_{2}$. WT pollen tubes that ruptured exhibited first an increase in the cytoplasmic calcium signal at the apex of the pollen tube (Figure 4A, top row). The increase in signal then propagated down the shank while the signal at the apex remained elevated (Figure 4A). These observations are in agreement with what was described previously (Duan et al., 2014). In wit12 pollen tubes, a similar sequence of events was observed prior to burst, suggesting that there is no significant difference in the $\mathrm{H}_{2} \mathrm{O}_{2}$-induced tip $\mathrm{Ca}^{2+}$ response (Figure 4A, bottom row).

Next, we described the $\mathrm{Ca}^{2+}$ signature in the VN of WT and wit12 pollen in response to $\mathrm{H}_{2} \mathrm{O}_{2}$. Pollen tubes, expressing the NLS-YC3.6 sensor, were treated with $\mathrm{H}_{2} \mathrm{O}_{2}$ at 7 hpp. $\mathrm{A} \mathrm{Ca}^{2+}$ peak was defined as a signal that both increased and decreased at least 0.5 A.U., or that increased at least 0.5 A.U. immediately prior to rupture. A range of nuclear 
$\mathrm{Ca}^{2+}$ peaks were recorded, from a peak height of $7 \mathrm{~A} . \mathrm{U}$. to the lowest peak counted of 0.5 A.U. (Figures 4B, 4C). Within both populations, we observed pollen tubes that showed a peak either immediately prior to rupture (Figure 4B), a peak followed by a signal decline (Figure 4C), or no $\mathrm{Ca}^{2+}$ peak as defined here (Figure 4D). 60\% of WT pollen tubes that burst showed a peak, compared to $50 \%$ that did not burst (Figure 4E). $35 \%$ of wit12 pollen tubes that burst and $39 \%$ of wit12 pollen tubes that did not burst showed a peak (Figure 4E). Thus, overall fewer wit12 pollen tubes showed a post- $\mathrm{H}_{2} \mathrm{O}_{2}$ nuclear $\mathrm{Ca}^{2+}$ spike compared to WT, but this reduction was not correlated with whether the pollen tubes burst or did not burst.

Regardless of whether the pollen tube ruptured, $55 \%$ of WT pollen tubes had a $\mathrm{Ca}^{2+}$ spike (Figure 4F). When the wit12 population was split up based on the distance of the VN from the pollen tube tip, $40 \%$ of VNs positioned between $100 \mu \mathrm{m}$ and $200 \mu \mathrm{m}$ from the tip showed a $\mathrm{Ca}^{2+}$ spike, compared to only 33\% VNs located over $200 \mu \mathrm{m}$ from the tip (Figure 4F). In addition, of the 20 pollen tubes randomly chosen that did burst, 14 had the VN between $100 \mu \mathrm{m}$ and $200 \mu \mathrm{m}$ and only 6 over $200 \mu \mathrm{m}$ from the tip. Among the 23 non-bursting wit12 pollen tubes, the VN was over $200 \mu \mathrm{m}$ from the tip in 12 cases, again suggesting that the capacity to undergo $\mathrm{H}_{2} \mathrm{O}_{2}$-induced burst correlates with the position of the nucleus.

Together, these data suggest that there is a correlation between nuclear $\mathrm{Ca}^{2+}$ spiking and $\mathrm{VN}$ position, as well as between $\mathrm{H}_{2} \mathrm{O}_{2}$-induced burst and $\mathrm{VN}$ position, but there is no correlation between nuclear $\mathrm{Ca}^{2+}$ spiking in individual pollen tubes and the capacity of the specific pollen tube to rupture.

The relationship at the single-cell level between the $\mathrm{VN} \mathrm{Ca}^{2+}$ spiking and $\mathrm{ROS}$-induced pollen tube burst remains unclear. Nevertheless, this is the first evidence for nuclear $\mathrm{Ca}^{2+}$ spikes in a higher plant gametophyte, and in light of the known relevance of nuclear $\mathrm{Ca}^{2+}$ signaling in the sporophyte, likely functionally relevant. It is possible that the VN senses cytoplasmic $\mathrm{Ca}^{2+}$ signatures, including those elevated by ROS, but that the nuclear $\mathrm{Ca}^{2+}$ spikes trigger downstream signals unrelated to pollen tube burst. 
Newly developed dual $\mathrm{Ca}^{2+}$ sensors (Kelner et al., 2018) will be better equipped to resolve the timing relationship between cytoplasmic and nuclear $\mathrm{Ca}^{2+}$ signatures and the relationship between the spread of cytoplasmic $\mathrm{Ca}^{2+}$ in the shank and the position of the nucleus. Discovering and investigating male gametophyte-expressed nuclear envelope-associated $\mathrm{Ca}^{2+}$ channels can now address which processes are affected by disrupting $\mathrm{VN} \mathrm{Ca}{ }^{2+}$ signatures.

\section{Supplemental Data}

The following materials are available in the online version of this article.

Supplemental Table S1: Primers used for cloning. CACC sites for directional TOPO cloning are indicated in bold. Specific recognition sites for Sacl and Spel are underlined.

\section{Supplemental Movie S1}

An example of cytoplasmic $\mathrm{Ca}^{2+}$ signatures in a WT pollen tube after addition of $\mathrm{H}_{2} \mathrm{O}_{2}$. The $\mathrm{Ca}^{2+}$ sensor R-GECO1 (Lat52pro::R-GECO1) was used to measure changes in $\mathrm{Ca}^{2+}$ over time. The pollen tube tip was imaged every 5 seconds until rupture. Movie playback speed is $60 \mathrm{x}$.

\section{Supplemental Movie S2}

An example of cytoplasmic $\mathrm{Ca}^{2+}$ signatures in a wit12 pollen tube after addition of $\mathrm{H}_{2} \mathrm{O}_{2}$. The $\mathrm{Ca}^{2+}$ sensor R-GECO1 (Lat52 pro::R-GECO1) was used to measure changes in $\mathrm{Ca}^{2+}$ over time. The pollen tube tip was imaged every 5 seconds until rupture. Movie playback speed is $60 \mathrm{x}$.

\section{Acknowledgements}

This work was funded by a National Science Foundation grant to I.M. (NSF-1613501). We would like to thank all members of the Meier lab for many fruitful discussions throughout this work. We thank Dr. Keith Slotkin (Donald Danforth Plant Science Center) for gifting the LAT52 pro::GFP construct, Dr. Melanie Krebs (Universität Heidelberg) for gifting UBQ10 pro::NLS-YC3.6, Dr. Simon Gilroy (University of Wisconsin- 
Madison) for gifting 35S pro::YC3.6 and Dr. Anna Dobritsa (The Ohio State University) for critically reading this manuscript.

\section{Materials and Methods}

\section{Plant materials}

Arabidopsis thaliana (Columbia-0 ecotype) was germinated on Murashige and Skoog medium plates (Caisson Laboratories) containing 1\% sucrose under constant light. Plants at the two-leaf stage were transplanted to soil and grown at an average temperature of $22-23^{\circ} \mathrm{C}$ under a 16-hour light/8-hour dark regime. The wit1-1 (GABI-Kat 470E06) wit2-1 (SALK_127765) (wit12) double null mutant was reported by (Zhao et al., 2008) and the quintuple null mutant wip1-1 (SAIL_390_A08) wip2-1 (SALK_052226) wip3-1 (GABI-Kat 459H07) wit1-1 wit2-1 (“wifi”) mutant was reported by (Zhou et al., 2012). Heterozygous male sterility-1 ( $m s-1$ ) plants were obtained from the Arabidopsis Biological Resource Center (https://abrc.osu.edu/).

\section{Cloning}

All primers used in cloning and construct generation are outlined in Supplemental Table 1. The pollen specific promoter LAT52 was cloned from the LAT52 pro::GFP construct in the binary plasmid pMDC107 as previously reported (Zhou and Meier, 2014). Restriction sites for enzymes Sacl and Spel were added to the 5' and 3' ends respectively. The amplified fragment was digested with the appropriate restriction enzymes. The LAT52 promoter fragment was isolated, purified with the QIAquik PCR Purification kit (Qiagen) and subsequently ligated into a pH2GW7 vector (Takagi et al., 2011).

The Yellow Cameleon 3.6 (YC3.6) calcium $\left(\mathrm{Ca}^{2+}\right)$ sensor N-terminally tagged with a nuclear localization signal (NLS) was cloned from a UBQ10 pro::NLS-YC3.6 (Krebs et al., 2011). The R-GECO1 $\mathrm{Ca}^{2+}$ sensor construct was amplified from $C M V_{\text {pro:::R-GECO1 }}$ (Zhao et al., 2011). NLS-YC3.6 and R-GECO1 were cloned into pENTR/D-TOPO

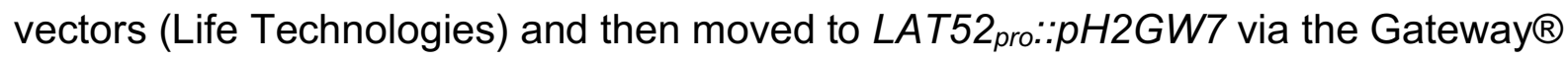
$\mathrm{LR}$ reaction (Life Technologies). 


\section{Generation of transgenic plants}

Binary vectors containing $\mathrm{Ca}^{2+}$ sensors were transformed into either Agrobacterium tumefaciens strains ABI or GV3101 by triparental mating (Wise et al., 2006).

Arabidopsis plants were transformed using the Agrobacterium-mediated floral dip method (Clough and Bent, 1998). The Col-0 ecotype (WT), wit12 and wifi backgrounds were used for the transformation. Transgenic plants were isolated on MS plates supplemented with using $30 \mu \mathrm{g} / \mathrm{mL}$ hygromycin, and positive transformants were confirmed by confocal microscopy. Homozygous T2 plants were used for all assays described here.

\section{Semi in-vivo pollen germination and ROS-induced pollen tube rupture}

Pollen germination media (PGM) containing $5 \mathrm{mM} \mathrm{KCl}, 5 \mathrm{mM} \mathrm{CaCl}_{2}, 1 \mathrm{mM} \mathrm{Ca}\left(\mathrm{NO}_{3}\right)_{2}$, $1 \mathrm{mM} \mathrm{MgSO}_{4}, 10 \%$ sucrose, $0.01 \%$ boric acid, pH 7.5 (Palanivelu and Preuss, 2006) was heated with $0.4 \%(\mathrm{w} / \mathrm{v})$ agarose, pipetted onto a glass slide, and allowed to solidify before use. Anthers at time of anthesis were used to pollinate stigmas of male sterility 1 (ms-1) flowers at developmental stage 14 (Zhou and Meier, 2014). Two hours after pollination, stigmas were excised and placed horizontally onto the solid PGM agar pad. The stigmas were incubated for an additional 3 or 5 hours in a humidity chamber (Palanivelu and Preuss, 2006). Elongating pollen tubes were imaged using a 4X objective with 5 times digital zoom. At time of imaging, pollen tubes were treated with $10 \mu \mathrm{L}$ of liquid PGM (control) or with $10 \mu \mathrm{L}$ of liquid PGM containing $6 \mathrm{mM} \mathrm{H}_{2} \mathrm{O}_{2}$.

Immediately following treatment, single plane images of pollen tubes were acquired every 10 seconds for 10 minutes with confocal microscopy (Eclipse C90i; Nikon). In addition, after this 10-minute time course, images were acquired at different focal planes to ensure that all pollen tubes could be identified and counted. To quantify the percentage of rupture events, the total number of intact and germinated pollen tubes were tabulated prior to and 10 minutes after treatment. The number of pollen tube discharge events were then counted. For the $\mathrm{Ca}^{2+}$ depletion experiments, pollen was germinated on low-Ca ${ }^{2+} \mathrm{PGM}$ medium $\left(5 \mathrm{mM} \mathrm{KCl}, 100 \mu \mathrm{M} \mathrm{CaCl}_{2}, 1 \mathrm{mM} \mathrm{MgSO}_{4}, 10 \%\right.$ sucrose, $0.01 \%$ boric acid, $\mathrm{pH} 7.5$ ). Pollen tubes were then treated with $10 \mu \mathrm{L}$ of low- 
$\mathrm{Ca}^{2+}$ PGM containing $100 \mu \mathrm{M} \mathrm{GdCl} 3$ and after a 10 -minute incubation $10 \mu \mathrm{L}$ of low-Ca ${ }^{2+}$ PGM with $12 \mathrm{mM} \mathrm{H}_{2} \mathrm{O}_{2}$ was added.

\section{Time to rupture quantification}

The time to rupture was quantified using data collected in Figure 1D. For each experiment, the 10-minute videos were split into 1-minute intervals and the number of pollen tubes that burst during each interval was recorded. That number was then divided by the total number of pollen tubes that ruptured over the course of the 10minute video and represented as percent. The average percent rupture for each time point was plotted.

\section{Measurement of positions of male germ unit components within the pollen tube}

WT and wit12 lines expressing both the VN marker Ub10 pro::NLS-mCherry and SC marker $M G H 3_{\text {pro:: }}$ GFP were germinated using the semi-in vivo method on PGM agar pads, as described above. Elongated pollen tubes were imaged using a 40X water immersion objective. Images of pollen at 5 and $7 \mathrm{hpp}$ were acquired at several different focal planes to ensure the MGU and pollen tube tip were in focus. Distances from the relative center of the sperm cells (SCs) and vegetative nucleus (VN) to the tip of the pollen tube were measured using the NIS Elements analysis software (Nikon).

\section{$\mathrm{Ca}^{2+}$ sensor imaging}

Pollen expressing either the R-GECO1 $\mathrm{Ca}^{2+}$ sensor or the NLS-YC3.6 nuclear $\mathrm{Ca}^{2+}$ sensor was germinated as previously described (Boavida and McCormick, 2007). A square was drawn onto a glass slide with a grease pencil (Staples). An isolated, pollinated stigma was placed in the center of the square. The square was filled with liquid PGM until the media began to mound. The slide was placed in a high humidity chamber for 5 hours to allow pollen tube elongation (Boavida and McCormick, 2007; Johnson and Kost, 2010). Individual elongating pollen tubes were imaged under a coverslip with a 40X water immersion objective with $2 x$ digital zoom. NLS-YC3.6 sensor was excited with $457 \mathrm{~nm}$, and fluorescence emission was detected between 465 and $505 \mathrm{~nm}$ (CFP) and between $530 \mathrm{~nm}$ and 570 (cpVenus). R-GECO1 was excited with 
$561 \mathrm{~nm}$, and its emission was detected between 620 and $650 \mathrm{~nm}$. Time lapse movies of the pollen tube tip and VN were generated by acquiring an image every 3 seconds for a total of 10 minutes. Because expression levels were highly variable between transgenic lines and pollen tubes, laser and gain settings were adjusted individually to obtain comparable baseline intensity values for each experiment. To quantify the $\mathrm{Ca}^{2+}$ signature, a region of interest (ROI) was defined using the binary editor function of the NIS Elements analysis software. For cytoplasmic $\mathrm{Ca}^{2+}$, an $\mathrm{ROI}$ was drawn proximal to the pollen tube tip. For nuclear $\mathrm{Ca}^{2+}$, an ROI was drawn around the VN. The mean fluorescence intensity was obtained for the defined ROI for every image captured in the time-lapse dataset and graphed as a function of time ("Calcium Signature"). For NLSYC3.6, the YFP/CFP ratio for each time point was calculated by dividing the mean fluorescence intensity of YFP by the mean fluorescence intensity of CFP.

\section{Peak number and standard deviation measurement}

$\mathrm{Ca}^{2+}$ signatures during pollen tube growth were quantified based on the number of peaks and the standard deviation of the RFP signal for R-GECO1 and the YFP/CFP ratio for NLS-YC3.6. Cytoplasmic peaks were determined based on an RFP fluorescence intensity increase of 150 A.U. or higher from the baseline, while nuclear $\mathrm{Ca}^{2+}$ peaks were determined based on a YFP/CFP ratio increase of $0.25 \mathrm{~A}$.U. or higher. The average fluorescence intensity or YFP/CFP ratio and the standard deviation for each 200-frame time-lapse movie was calculated. All 20 standard deviations were then averaged to determine the mean standard deviation for R-GECO1 in WT, R-GECO1 in wit12, NLS-YC3.6 in WT, and NLS-YC3.6 in wit12. Nuclear $\mathrm{Ca}^{2+}$ signatures after addition of $\mathrm{H}_{2} \mathrm{O}_{2}$ were analyzed using a similar peak determination as described above. However, a nuclear $\mathrm{Ca}^{2+}$ peak was determined based on a YFP/CFP ratio increase of 0.50 A.U. or higher.

\section{Circularity index measurement}

WT and wit12 pollen tubes expressing Lat52 pro::NLS-YC3.6 were grown using the semiin vivo method and imaged at $7 \mathrm{hpp}$ with a confocal microscope (Eclipse C90i, Nikon). Images were taken using a 40X water immersion objective and acquired using NIS- 
Elements AR version 3.2. At least 150 pollen vegetative nuclei were visualized for each line by creating z-stack sections $(3 \mu \mathrm{m})$ to capture the entire nucleus. A maximum intensity projection of each nucleus was generated, and ImageJ was used to calculate the circularity index of each nucleus.

\section{Accession Numbers}

Sequence data from this article can be found in the GenBank/EMBL data libraries under accession numbers NM_001160797 (WIP1), NM_125004 (WIP2), NM_112181 (WIP3), NM_121177 (WIT1), and NM_105565 (WIT2).

\section{Figure Legends:}

Figure 1. $\mathrm{Ca}^{2+}$-dependent ROS-induced pollen tube burst correlates with nuclear position.

A-B, Semi-in vivo germinated WT pollen expressing Lat52 pro::R-GECO1 $\mathrm{Ca}^{2+}$ sensor. Size bars $=50 \mu \mathrm{m}$. A, Pollen tubes prior to incubation with the ROS generating compound $\mathrm{H}_{2} \mathrm{O}_{2}$. B, Pollen tubes following incubation with $\mathrm{H}_{2} \mathrm{O}_{2}$. Several tubes have ruptured as indicated by cytoplasm outside the pollen tube. The asterisks in $A$ and $B$ mark the same pollen tube before and after rupture. The white dotted line marks the base of the stigma. C-D, ROS induced pollen tube rupture in semi-in vivo germinated pollen at 5 hours (C) and 7 hours (D) post pollination (hpp). Values represent the average number of ruptured pollen tubes observed when treated with buffer, $\mathrm{H}_{2} \mathrm{O}_{2}$, or $\mathrm{H}_{2} \mathrm{O}_{2}$ and a Ca ${ }^{2+}$ channel inhibitor, gadolinium(III) chloride $\left(\mathrm{GdCl}_{3}+\mathrm{H}_{2} \mathrm{O}_{2}\right)$. Bars are standard error. $\mathrm{N}$ equals total number of pollen tubes. ${ }^{*} \mathrm{P} \leq 0.05 ;{ }^{* *} \mathrm{P} \leq 0.01 ;{ }^{* * *} \mathrm{P} \leq 0.001$ for Student's t-test; N.S. indicates no significance by Student's t-test. E, The time to ROS induced pollen tube rupture in semi-in vivo germinated WT and wit12 pollen at 7 hpp. Bars are standard error. $N$ equals total number of pollen tubes. ${ }^{*} \mathrm{P} \leq 0.05 ;{ }^{* *} \mathrm{P} \leq 0.01$; ${ }^{* * *} \mathrm{P} \leq 0.001$ for Student's t-test. F-I, The localization of the VN (orange) and SCs (green) was examined in elongating pollen tubes at 5 and $7 \mathrm{hpp}$. The VN and SCs were visualized using Ub10 pro::NLS-mCherry and MGH3pro::GFP respectively. The position of the VN tip in WT remains close to the pollen tube tip at 5 (F) and $7 \mathrm{hpp}(\mathbf{G})$. The VN in wit12 pollen increases in distance from the pollen tube tip over time. The nucleus is 
closer at $5 \mathrm{hpp}(\mathbf{H})$ than at $7 \mathrm{hpp}(\mathbf{I})$. Scale bars $=10 \mu \mathrm{m}$. J, The position of the SCs and VN relative to the tip and distance between the SCs and VN were quantified for WT and wit12 pollen tubes at both time points. $\mathrm{N}=150$ pollen tubes. Bars are standard error. ${ }^{* *} \mathrm{P} \leq 0.01 ;{ }^{* * *} \mathrm{P} \leq 0.001$ for Student's t-test. K-L, Representative images of a WT VN (K) and a wit12 VN (L) at $7 \mathrm{hpp}$, using the YFP signal of NLS-YC3.6 as a nuclear marker. M, Circularity index of vegetative nuclei from WT and wit12 pollen tubes. (Left) Scatter plot showing each nucleus as a single point. (Right) Box plot. (Top line) Maximum. (Box) Quartiles. (Solid middle line) Median. (Dotted middle line) Mean. (Bottom line) Bottom fence. ${ }^{* *} \mathrm{P}<0.001$ for Student's t-test.

Figure 2. Cytoplasmic $\mathrm{Ca}^{2+}$ fluctuations are not disrupted in wit12 pollen tubes. A-F, The cytoplasmic $\mathrm{Ca}^{2+}$ sensor R-GECO1 (Lat52pro::R-GEC01) was used to measure $\mathrm{Ca}^{2+}$ signatures in elongating pollen tubes. A-B, Images of changes in cytoplasmic $\mathrm{Ca}^{2+}$ fluctuations during growth for a WT pollen tube (A) and a wit12 pollen tube (B). Images in $\mathbf{A}$ and $\mathbf{B}$ correspond to time points 1 and 2, shown in $\mathbf{C}$ and $\mathbf{D}$. Scale bar $=10 \mu \mathrm{m}$. C, Cytoplasmic $\mathrm{Ca}^{2+}$ fluctuations measured at the pollen tube tip of WT pollen are represented as fluorescence intensity in the blue line. The red line marks the pollen tube growth from the origin at the start of imaging. The black line represents a 4-point rolling average of the data used to highlight the overall trend. Numbers in the graph correspond to the image numbers in A. D, Cytoplasmic $\mathrm{Ca}^{2+}$ fluctuations at the tip of elongating wit12 pollen. Fluorescence intensity is depicted by the red line. The blue line marks the pollen tube growth from the origin at the start of imaging. The black line represents a 4-point rolling average of the data used to highlight the overall trend.

Numbers in the graph correspond to the image numbers in B. E-F, Cytoplasmic $\mathrm{Ca}^{2+}$ signatures were quantified based on the number of peaks $(\mathbf{E})$ and the standard deviation of the fluorescence intensity for the entire time-lapse movie (F). 20 pollen tubes were analyzed in each case. Bars are standard error. N.S. indicates no significant difference by Student's t-test.

Figure 3. Pollen nuclear $\mathrm{Ca}^{2+}$ signatures correlate with the position of the nucleus 
A-G, The nucleus-localized $\mathrm{Ca}^{2+}$ sensor (Lat52pro::NLS-YC3.6) was used to measure $\mathrm{Ca}^{2+}$ signatures in the VNs of elongating pollen tubes A-C, Ratio images of changes in nuclear $\mathrm{Ca}^{2+}$ fluctuations during growth for a WT pollen tube with the VN positioned 60 $\mu \mathrm{m}$ from the tip (A), a wit12 pollen tube with the VN positioned $152 \mu \mathrm{m}$ from the tip (B), a wit12 pollen tube with the VN positioned $396 \mu \mathrm{m}$ from the tip (C). Scale bar $=10 \mu \mathrm{m}$. D, A representative nuclear $\mathrm{Ca}^{2+}$ signature for a WT nucleus $60 \mu \mathrm{m}$ from the pollen tube tip, presented as FRET ratio of YFP to CFP fluorescence intensity, is shown as a blue line. Numbers in the graph correspond to the image numbers in $\mathbf{A}$. E, A representative nuclear $\mathrm{Ca}^{2+}$ signature for a wit12 nucleus $152 \mu \mathrm{m}$ from the pollen tube tip, presented as a FRET ratio of YFP to CFP fluorescence intensity, is shown as a red line. A representative nuclear $\mathrm{Ca}^{2+}$ signature for a wit12 nucleus $396 \mu \mathrm{m}$ from then pollen tube tip, presented as a FRET ratio of YFP to CFP fluorescence intensity, is shown as a black line. Numbers in the graph correspond to the image numbers in $\mathbf{B}$ and $\mathbf{C}$, respectively. F-G, Nuclear $\mathrm{Ca}^{2+}$ signatures were quantified based on the number of peaks (F) and the standard deviation of the YFP/CFP ratio for the entire time-lapse movie (G). At least 20 pollen tubes were analyzed per background. Columns were split based on the distance of the VN from the pollen tube tip. Bars are standard error. * $\mathrm{P}<0.05 ;{ }^{* *} \mathrm{P}<0.01 ;{ }^{* * *} \mathrm{P}<0.001$.

\section{Figure 4. Post-ROS $\mathrm{Ca}^{2+}$ fluctuations}

A, The $\mathrm{Ca}^{2+}$ sensor R-GECO1 (Lat52pro::R-GECO1) was used to measure cytoplasmic $\mathrm{Ca}^{2+}$ signatures over time in pollen tubes after addition of $\mathrm{H}_{2} \mathrm{O}_{2}$. Representative images of changes in cytoplasmic $\mathrm{Ca}^{2+}$ fluctuations for one WT pollen tube (top panel) and one wit12 pollen tube (bottom panel). The white dotted line outlines the pollen tube wall using the corresponding DIC image. Numbers in the bottom left corner are the RFP fluorescence of the corresponding ROls (yellow circles), located close to the pollen tube tip. Time 0 indicates the start of imaging after $\mathrm{H}_{2} \mathrm{O}_{2}$ addition. Imaging sequences shown are representative of several videos for each genotype. Scale bar $=10 \mu \mathrm{m}$. B-F, The $\mathrm{Ca}^{2+}$ sensor Lat52 pro::NLS-YC3.6 was used to measure nuclear $\mathrm{Ca}^{2+}$ signatures over time in the VNs after addition of $\mathrm{H}_{2} \mathrm{O}_{2}$. B-D, Examples of the different types of nuclear $\mathrm{Ca}^{2+}$ signatures observed in WT after addition of $\mathrm{H}_{2} \mathrm{O}_{2}$. A range of nuclear $\mathrm{Ca}^{2+}$ spikes 
are shown, from the highest peak height of 7 A.U. (B) to the lowest peak height of 0.5 A.U. (C). D, A nuclear $\mathrm{Ca}^{2+}$ signature without a spike. E-F, Quantification of presence or absence of peaks for pollen tubes that burst and pollen tubes that fail to burst for both WT and wit12. The numbers of pollen tubes with or without a peak for each condition is shown in the bar graph. E, WT is compared to the entire wit12 population. The percent of pollen tubes that presented a nuclear $\mathrm{Ca}^{2+}$ peak is shown above each bar. $\mathbf{F}$, wit12 columns were split based on the distance of the VN from the pollen tube tip. The percent of pollen tubes that presented a nuclear $\mathrm{Ca}^{2+}$ peak, with burst and no burst combined for each group, is shown above each bar.

\section{References}

Boavida LC, McCormick S (2007) Temperature as a determinant factor for increased and reproducible in vitro pollen germination in Arabidopsis thaliana. Plant J 52: 570-582

Boisson-Dernier A, Roy S, Kritsas K, Grobei MA, Jaciubek M, Schroeder JI, Grossniklaus U (2009) Disruption of the pollen-expressed FERONIA homologs ANXUR1 and ANXUR2 triggers pollen tube discharge. Development 136: 3279-3288

Borg M, Brownfield L, Twell D (2009) Male gametophyte development: a molecular perspective. J Exp Bot 60: 1465-1478

Capoen W, Sun J, Wysham D, Otegui MS, Venkateshwaran M, Hirsch S, Miwa H, Downie JA, Morris RJ, Ané J-M, Oldroyd GED (2011) Nuclear membranes control symbiotic calcium signaling of legumes. Proc Natl Acad Sci USA 108: 14348

Charpentier M (2018) Calcium signals in the plant nucleus: origin and function. J Exp Bot 69: 4165-4173

Charpentier M, Sun J, Martins TV, Radhakrishnan GV, Findlay K, Soumpourou E, Thouin J, Véry A-A, Sanders D, Morris RJ, Oldroyd GED (2016) Nuclear-localized cyclic nucleotide-gated channels mediate symbiotic calcium oscillations. Science 352: 1102

Clough SJ, Bent AF (1998) Floral dip: a simplified method for Agrobacterium-mediated transformation of Arabidopsis thaliana. Plant J 16: 735-743

Del Bene F, Wehman AM, Link BA, Baier H (2008) Regulation of neurogenesis by interkinetic nuclear migration through an apical-basal notch gradient. Cell 134: 1055-1065

Dresselhaus T, Franklin-Tong N (2013) Male-female crosstalk during pollen germination, tube growth and guidance, and double fertilization. Mol Plant 6: 1018-1036

Duan Q, Kita D, Johnson EA, Aggarwal M, Gates L, Wu H-M, Cheung AY (2014) Reactive oxygen species mediate pollen tube rupture to release sperm for fertilization in Arabidopsis. Nat Commun 5: 3129

Dumas C, Knox R, Gaude T (1985) The spatial association of the sperm cells and vegetative nucleus in the pollen grain of Brassica. Protoplasma 124: 168-174

Escobar-Restrepo J-M, Huck N, Kessler S, Gagliardini V, Gheyselinck J, Yang W-C, Grossniklaus U (2007) The FERONIA Receptor-like Kinase Mediates Male-Female Interactions During Pollen Tube Reception. Science 317: 656

Eyal Y, Curie C, McCormick S (1995) Pollen specificity elements reside in 30 bp of the proximal promoters of two pollen-expressed genes. Plant Cell 7: 373-384

Ge Z, Bergonci T, Zhao Y, Zou Y, Du S, Liu MC, Luo X, Ruan H, García-Valencia LE, Zhong S, Hou S, Huang Q, Lai L, Moura DS, Gu H, Dong J, Wu HM, Dresselhaus T, 
Xiao J, Cheung AY, Qu LJ (2017) pollen tube integrity and sperm release are regulated by RALF-mediated signaling. Science 358: 1596-1600

Graumann K, Runions J, Evans DE (2010) Characterization of SUN-domain proteins at the higher plant nuclear envelope. Plant J 61: 134-144

Higashiyama T, Yang W-c (2017) Gametophytic Pollen Tube Guidance: Attractant Peptides, Gametic Controls, and Receptors. Plant Physiol 173: 112

Iwano M, Entani T, Shiba H, Kakita M, Nagai T, Mizuno H, Miyawaki A, Shoji T, Kubo K, Isogai A, Takayama S (2009) Fine-Tuning of the Cytoplasmic $\mathrm{Ca}(2+)$ Concentration Is Essential for Pollen Tube Growth. Plant Physiol 150: 1322-1334

Iwano M, Ngo QA, Entani T, Shiba H, Nagai T, Miyawaki A, Isogai A, Grossniklaus U, Takayama S (2012) Cytoplasmic Ca2+ changes dynamically during the interaction of the pollen tube with synergid cells. Development 139: 4202-4209

Johnson MA, Harper JF, Palanivelu R (2019) A Fruitful Journey: Pollen Tube Navigation from Germination to Fertilization. Annu Rev Plant Biol 70: 809-837

Johnson MA, Kost B (2010) Pollen Tube Development. Methods Mol Biol 655: 155-176

Kelner A, Leitão N, Chabaud M, Charpentier M, de Carvalho-Niebel F (2018) Dual Color Sensors for Simultaneous Analysis of Calcium Signal Dynamics in the Nuclear and Cytoplasmic Compartments of Plant Cells. Front Plant Sci 9: 245

Kessler SA, Grossniklaus U (2011) She's the boss: signaling in pollen tube reception. Curr Opin Plant Biol 14: 622-627

Krebs M, Held K, Binder A, Hashimoto K, Den Herder G, Parniske M, Kudla J, Schumacher K (2011) FRET-based genetically encoded sensors allow high-resolution live cell imaging of Ca2+ dynamics. Plant J 69: 181-192

Lecourieux D, Lamotte O, Bourque S, Wendehenne D, Mazars C, Ranjeva R, Pugin A (2005) Proteinaceous and oligosaccharidic elicitors induce different calcium signatures in the nucleus of tobacco cells. Cell Calcium 38: 527-538

Leitão N, Dangeville P, Carter R, Charpentier M (2019) Nuclear calcium signatures are associated with root development. Nat Commun 10: 4865

Leydon AR, Beale KM, Woroniecka K, Castner E, Chen J, Horgan C, Palanivelu R, Johnson MA (2013) Three MYB transcription factors control pollen tube differentiation required for sperm release. Curr Biol 23: 1209-1214

Li H-J, Meng J-G, Yang W-C (2018) Multilayered signaling pathways for pollen tube growth and guidance. Plant Reprod 31: 31-41

Liang Y, Tan ZM, Zhu L, Niu QK, Zhou JJ, Li M, Chen LQ, Zhang XQ, Ye D (2013) MYB97, MYB101 and MYB120 function as male factors that control pollen tube-synergid interaction in Arabidopsis thaliana fertilization. PLoS Genet 9: e1003933

Liang Y, Tóth K, Cao Y, Tanaka K, Espinoza C, Stacey G (2014) Lipochitooligosaccharide recognition: an ancient story. New Phytol 204: 289-296

Mazars C, Bourque S, Mithöfer A, Pugin A, Ranjeva R (2009) Calcium homeostasis in plant cell nuclei. New Phytol 181: 261-274

McCue AD, Cresti M, Feijó JA, Slotkin RK (2011) Cytoplasmic connection of sperm cells to the pollen vegetative cell nucleus: potential roles of the male germ unit revisited. J Exp Bot 62: 1621-1631

Ngo QA, Vogler H, Lituiev DS, Nestorova A, Grossniklaus U (2014) A calcium dialog mediated by the FERONIA signal transduction pathway controls plant sperm delivery. Dev Cell 29: 491-500

Palanivelu R, Preuss D (2006) Distinct short-range ovule signals attract or repel Arabidopsis thaliana pollen tubes in vitro. BMC Plant Biol 6: 7

Pauly N, Knight MR, Thuleau P, van der Luit AH, Moreau M, Trewavas AJ, Ranjeva R, Mazars C (2000) Control of free calcium in plant cell nuclei. Nature 405: 754-755 
Russell S, Cass D (1981) Ultrastructure of the sperms of Plumbago zeylanica 1. Cytology and association with the vegetative nucleus. Protoplasma 107: 85-107

Starr DA, Fridolfsson HN (2010) Interactions between nuclei and the cytoskeleton are mediated by SUN-KASH nuclear-envelope bridges. Annu Rev Cell Dev Biol 26: 421-444

Takagi S, Islam MS, Iwabuchi K (2011) Dynamic Behavior of Double-Membrane-Bounded Organelles in Plant Cells. Int Rev Cell Mol Biol 286: 181-222

Tamura K, Iwabuchi K, Fukao Y, Kondo M, Okamoto K, Ueda H, Nishimura M, HaraNishimura I (2013) Myosin XI-i Links the Nuclear Membrane to the Cytoskeleton to Control Nuclear Movement and Shape in Arabidopsis. Curr Biol 23: 1776-1781

Wise AA, Liu Z, Binns AN (2006) Three Methods for the Introduction of Foreign DNA into Agrobacterium. Methods Mol Biol 343: 43-54

Zhang J, Huang Q, Zhong S, Bleckmann A, Huang J, Guo X, Lin Q, Gu H, Dong J, Dresselhaus T, Qu LJ (2017) Sperm cells are passive cargo of the pollen tube in plant fertilization. Nat Plants 3: 17079

Zhao Q, Brkljacic J, Meier I (2008) Two distinct interacting classes of nuclear envelopeassociated coiled-coil proteins are required for the tissue-specific nuclear envelope targeting of Arabidopsis RanGAP. Plant Cell 20: 1639-1651

Zhao Y, Araki S, Wu J, Teramoto T, Chang YF, Nakano M, Abdelfattah AS, Fujiwara M, Ishihara T, Nagai T, Campbell RE (2011) An expanded palette of genetically encoded $\mathrm{Ca}^{2+}$ indicators. Science 333: 1888-1891

Zhou X, Graumann K, Evans DE, Meier I (2012) Novel plant SUN-KASH bridges are involved in RanGAP anchoring and nuclear shape determination. J Cell Biol 196: 203-211

Zhou X, Meier I (2014) Efficient plant male fertility depends on vegetative nuclear movement mediated by two families of plant outer nuclear membrane proteins. Proc Natl Acad Sci USA 111: 11900-11905 
Figure 1
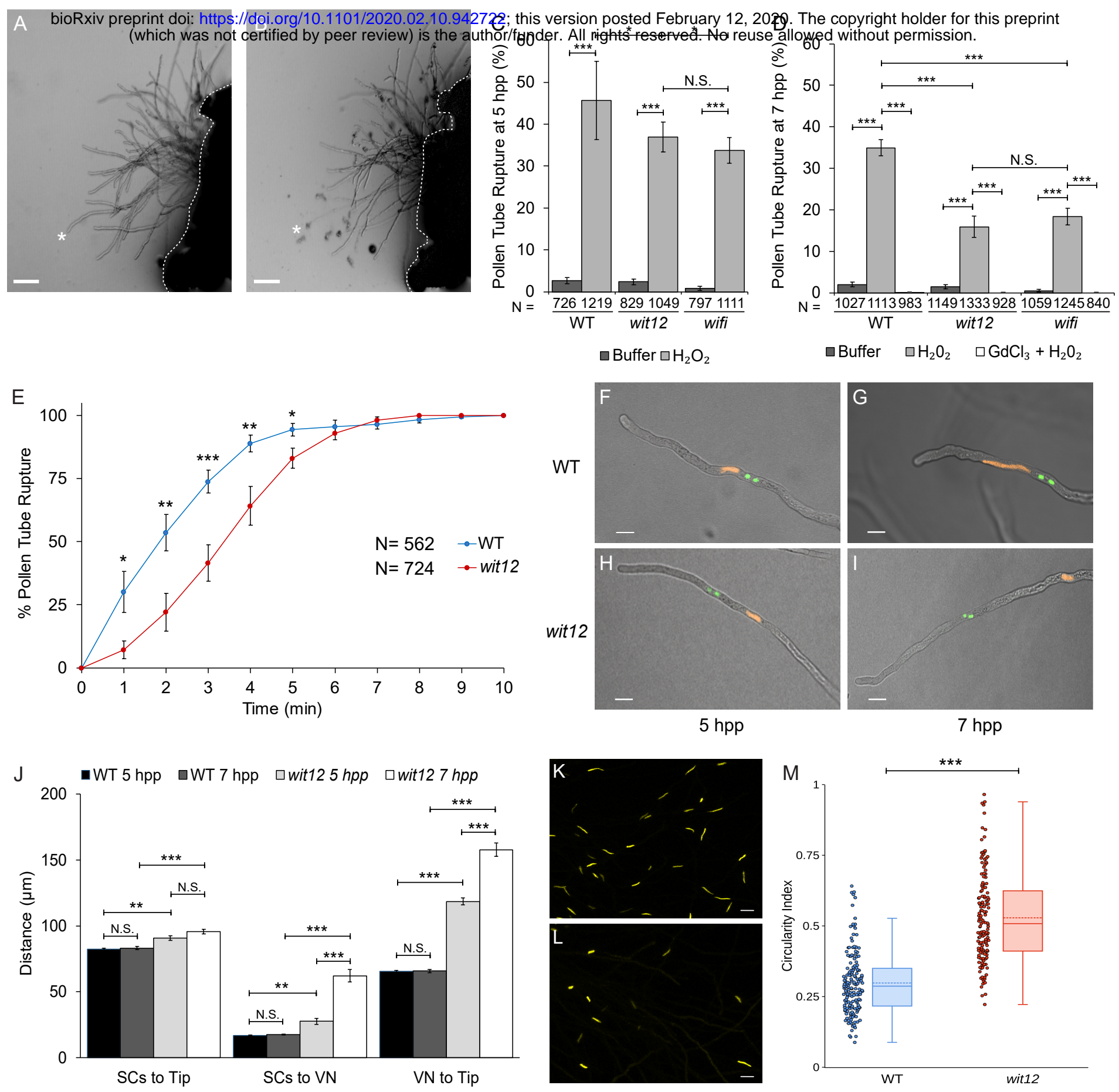

5 hpp

7 hpp

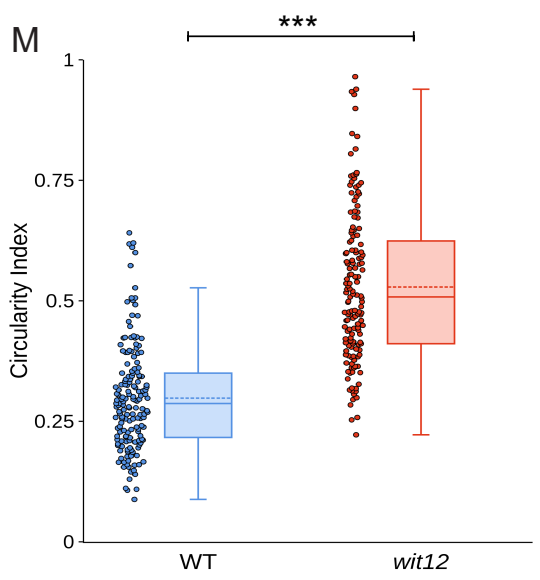


Figure 2

bioRxiv preprint doi: https://doi.org/10.1101/2020.02.10.942722; this version posted February 12, 2020. The copyright holder for this preprint

A (which was not certified by peer review) is the author/funder All rights reserved. No reuse allowed without permission.
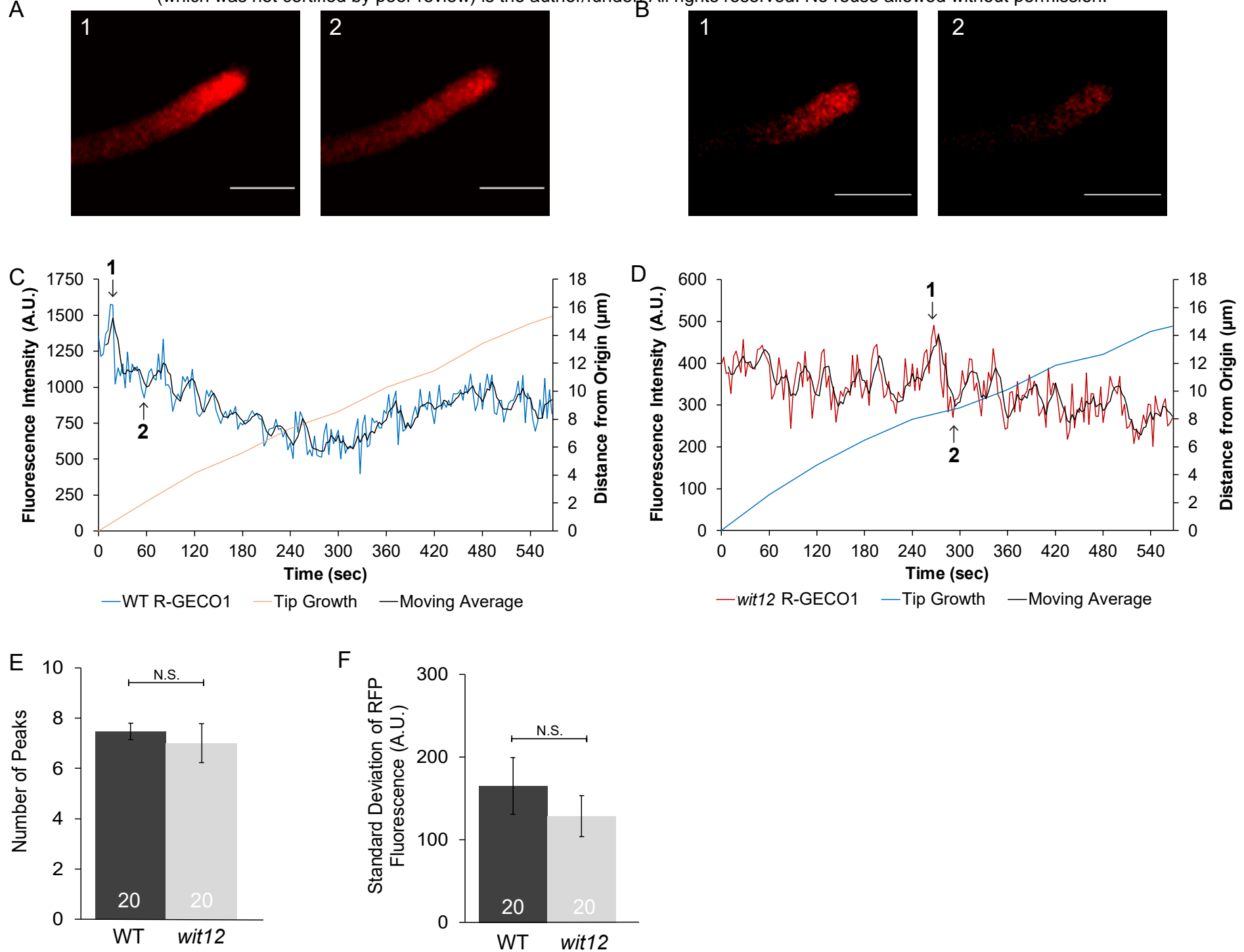
Figure 3

A

WT B

B wit12

C

wit12

bioRxivpreprint_doi: https://doi.org/10.1101/2020.02.10.942722; this versjon posted February 12, 2020. The copyjight holder for this preprint

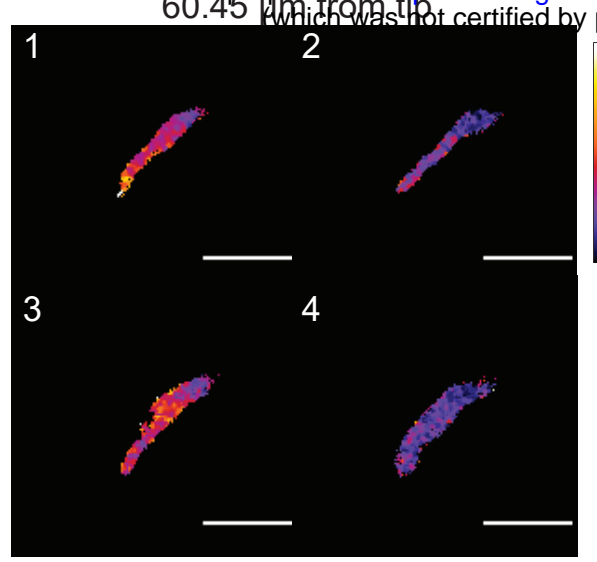

D
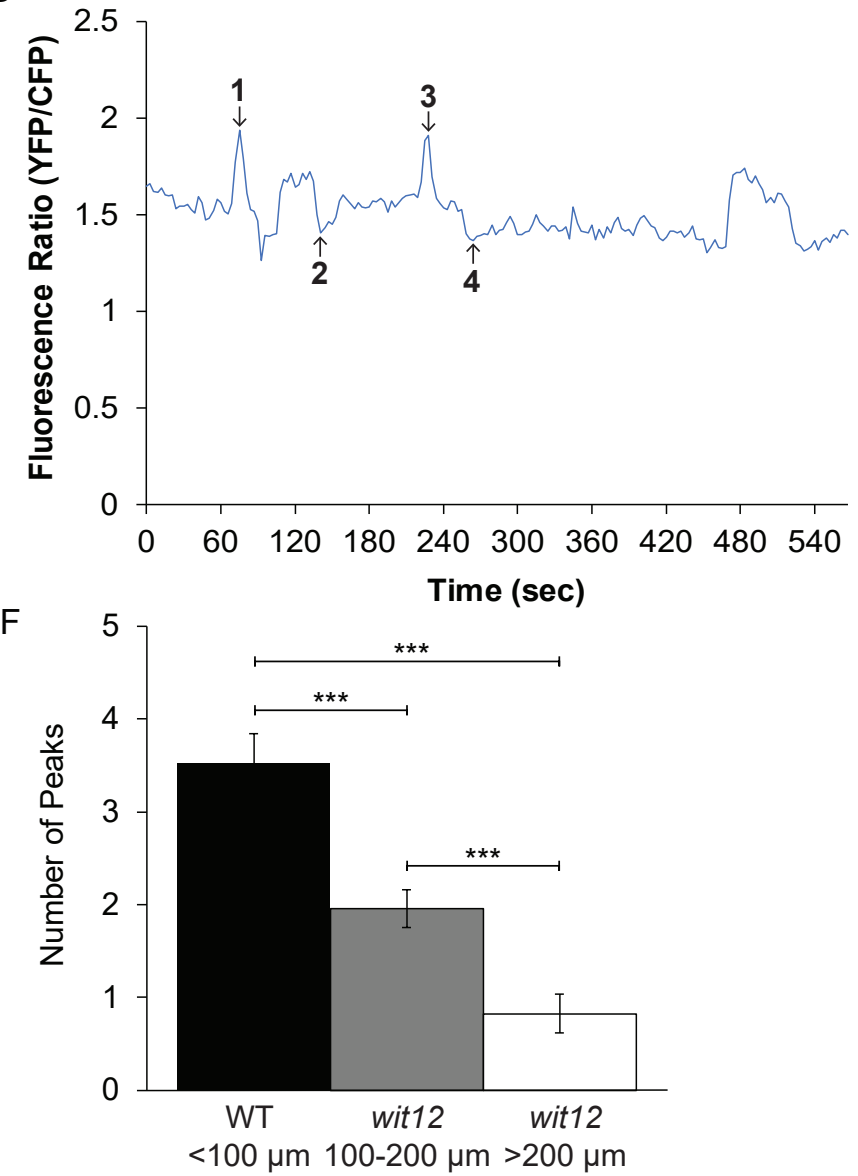

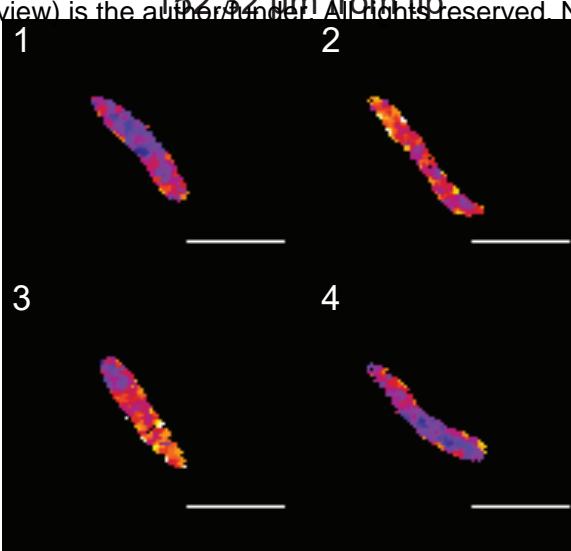

E
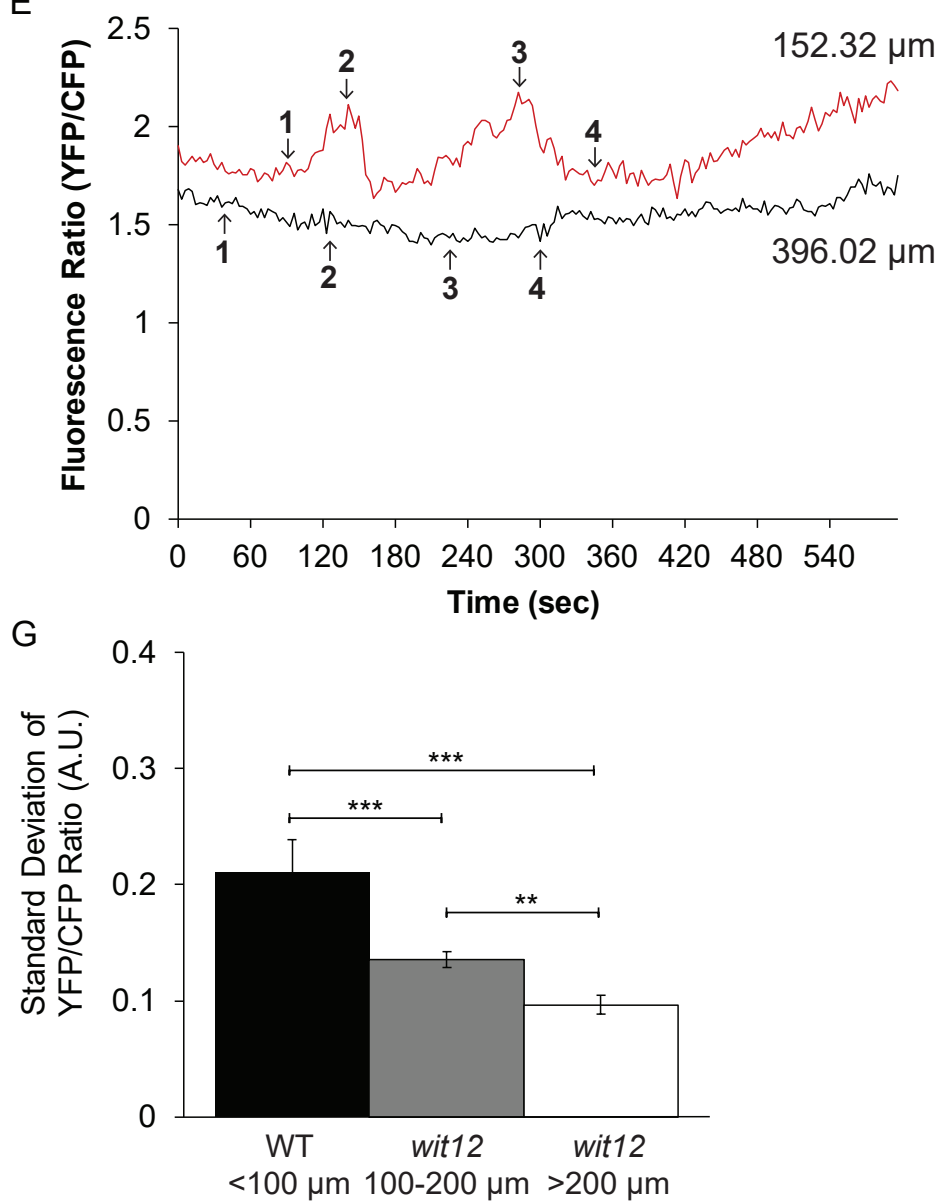
Figure 4 A

bioRxiv preprint doi: https://doi.org/10.1101/2020.02.10.942722; this version posted February 12, 2020. The copyright holder for this preprint (which was not certified by peer review) is the author/funder. All rights reserved. No reuse allowed without permission.
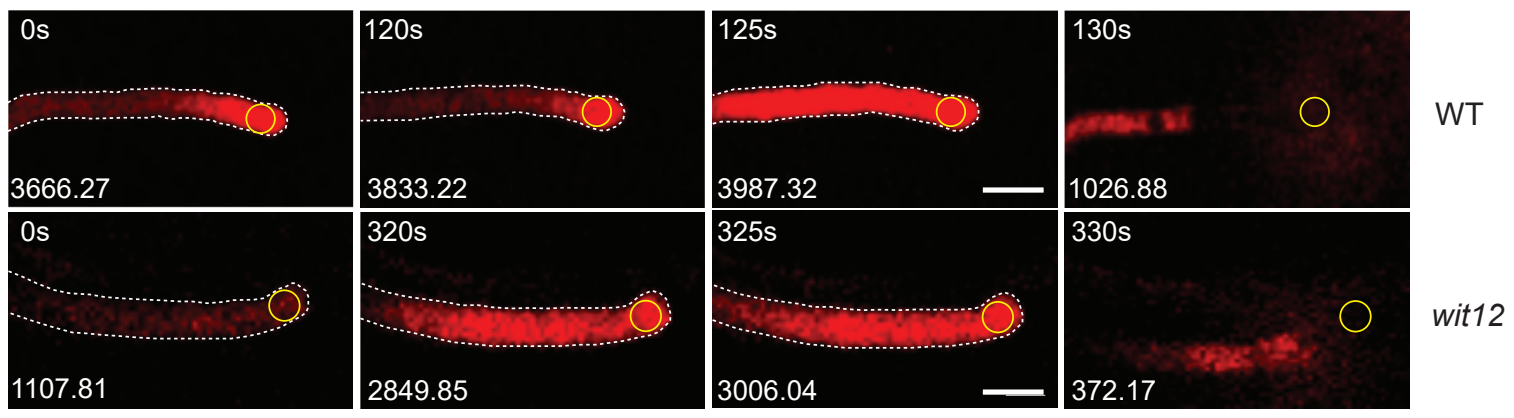

B

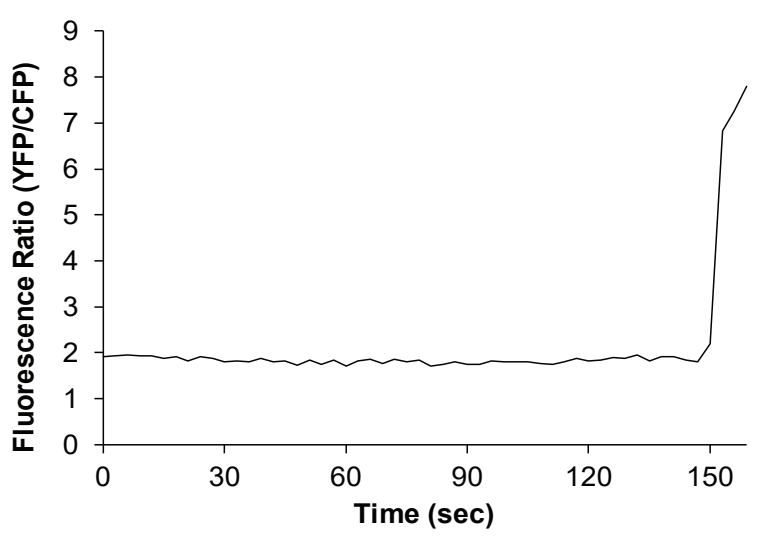

$\mathrm{D}$

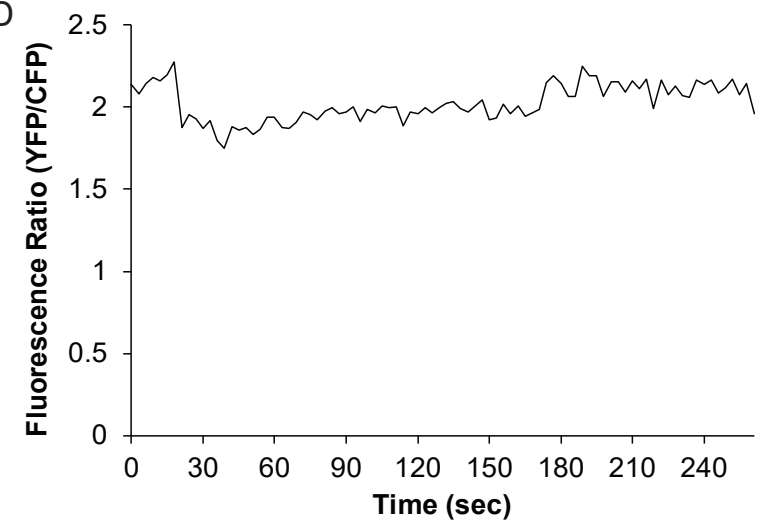

$\mathrm{F}$

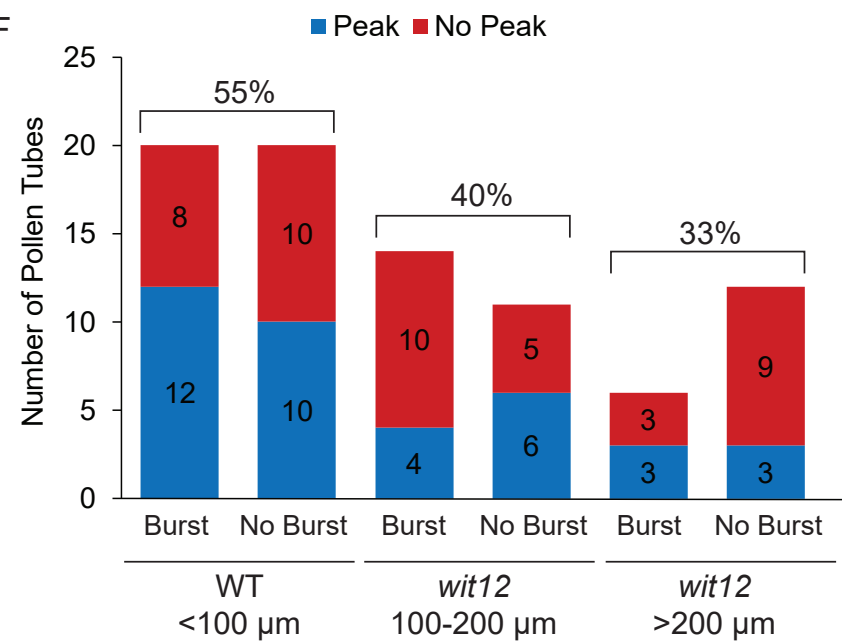

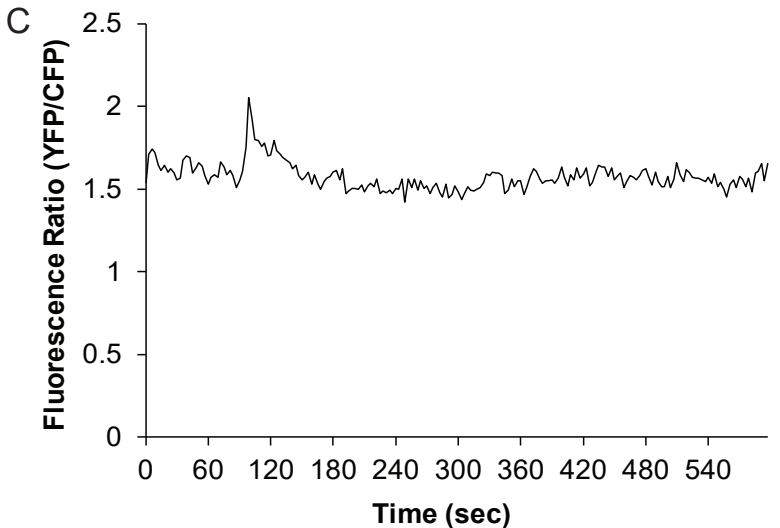

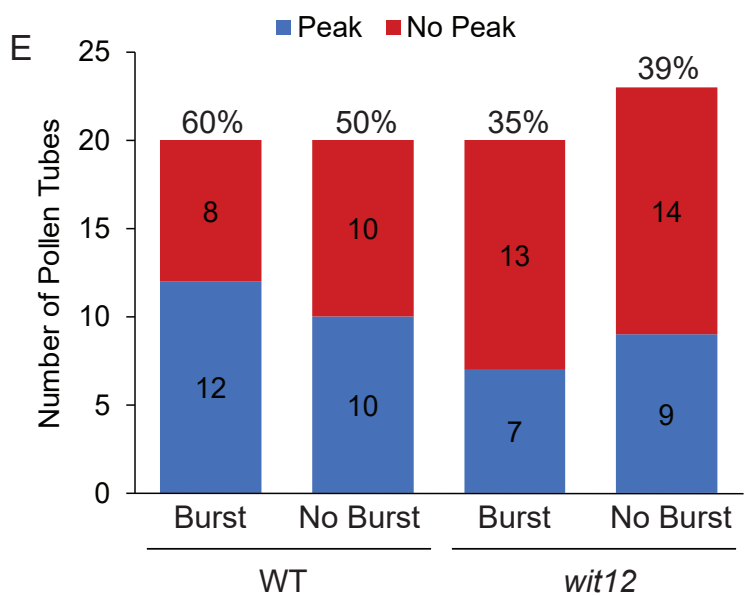

